\title{
Research Paper: Behavioral and Molecular Analysis of Antioxidative Potential of Rosmarinic Acid Against Methamphetamine-induced Augmentation of Casp3a mRNA in the Zebrafish Brain
}

Vida Nikshenas Shahrestani ${ }^{1} \odot$, Mohammad Haddadi ${ }^{*} \odot$, Ali Reza Samzadeh Kermani ${ }^{2} \odot$

1. Department of Biology, Faculty of Basic Sciences, University of Zabol, Zabol, Iran.

2. Department of Chemistry, Faculty of Basic Sciences, University of Zabol, Zabol, Iran.

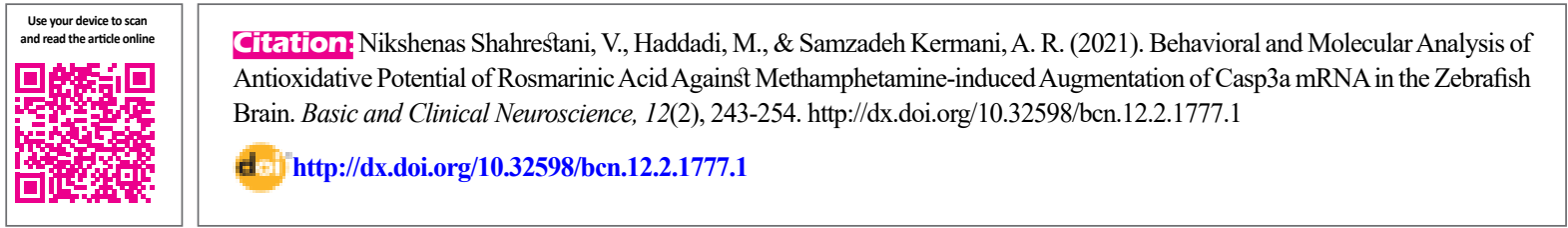

(A) 03

Article info:

Received: 10 Apr 2020

First Revision: 14 May 2020

Accepted: 01 Dec 2020

Available Online: 01 Mar 2021
Keywords:

Methamphetamine, Casp3a, Rosmarinic acid, Zinc oxide, Chitosan nanoparticle, Zebrafish, Behavioral test

\section{ABSTRACT}

Introduction: Methamphetamine (MA) acts as a powerful oxidant agent, while Rosmarinic Acid (RA) is an effective herbal antioxidant. Oxidative stress-mediated by MA results in apoptosis, and caspase- 3 is one of the critical enzymes in the apoptosis process. MA can epigenetically alter gene regulation. In this paper, to investigate the effects of RA on MA-mediated oxidative stress, changes in the level of casp3a mRNA were demonstrated in zebrafish.

Methods: The animals were grouped in 3 treatment conditions for the behavioral test: control, MA, MA pretreated by RA, and 6 treatment conditions for the molecular test: control, RA, MA, MA co-treated with RA, MA co-treated with $\mathrm{RA} / \mathrm{ZnO} /$ chitosan nanoparticle, and $\mathrm{ZnO}$ / chitosan nanoparticle. Then molecular and behavioral investigations were carried out, and critical comparisons were made between the groups.

MA solution was prepared with a concentration of $25 \mathrm{mg} / \mathrm{L}$, and RA solution was prepared by DPPH test with the antioxidant power of about $97 \%$. Each solution was administered by immersing 20 zebrafish for 20 minutes, once per day for 7 days. The level of casp3a mRNA was quantified by using qRT-PCR. One-sided trapezoidal tank diving test was applied to study behavioral alterations.

Results: The qPCR analysis demonstrated the high potential of RA/ZnO/chitosan in counteracting the MA-mediated elevation in casp3a mRNA level. Based on the diving test results of MA-treated fish, MA was found to be anxiolytic compared to the control. While the resulted diving pattern of the MA-treated animals pretreated by RA was novel and different from both the control and MA-treated groups.

Conclusion: The potential of RA combined with a suitable nanoparticle against MA-induced oxidative stress was supported. The high efficiency of $\mathrm{ZnO} /$ chitosan in increasing RA penetration to the brain cells was evident. MA at a dose of $25 \mathrm{mg} / \mathrm{L}$ is anxiolytic for zebrafish. However, the molecular mechanisms involved in these processes should be studied. 


\section{Highlights}

- Methamphetamine (MA) induces excess casp3a transcript in the brain tissue in zebrafish.

- Immediate effect of chronic exposure to $25 \mathrm{mg} / \mathrm{L}$ MA is anxiolytic for zebrafish.

- Rosmarinic Acid (RA) counteracts the effects of MA-mediated oxidative stress.

- RA alone does not reduce the anxiolytic effects of MA and even enhances it.

- $\mathrm{ZnO} /$ chitosan nanoparticles increase RA penetration to the blood-brain barrier as fast as MA.

\section{Plain Language Summary}

Methamphetamine (MA) called ice or crystal in streets, is a drug of abuse. It has been associated with a variety of neurotoxic effects including oxidative stress, which leads to characteristic cell changes and death. The caspase-3 enzyme is one of the key enzymes of this process. There are storage forms of inactive Caspase- 3 in any cell called procaspase- 3 . It's been demonstrated previously that MA treatment causes activation of the procaspase- 3 resource. The present study was undertaken to investigate whether MA can change the regulation of Caspase- 3 gene expression and increase the synthesis of casp3a mRNA in zebrafish. qRT-PCR results demonstrated that MA increases the casp3a mRNA levels up to 18-fold compare to the control group. We were curious to know whether treatment by an antioxidant solution can counteract MA-mediated oxidative stress. So in the second part of this research, we investigated the herbal antioxidant molecule, Rosmarinic Acid (RA). Since the penetration of RA across the Blood-Brain Barrier is not as fast as MA, RA was combined with $\mathrm{ZnO} /$ chitosan nanoparticles and then provided to zebrafish as an animal model. The qRT-PCR analysis demonstrated the high potential of $\mathrm{RA} / \mathrm{ZnO} /$ chitosan in counteracting the MA-mediated elevation in the casp3a mRNA level and brought it back much closer to the level of the control group (2.8-fold). On the other hand, zebrafish is known as a non-mammalian anxiety model system. The immediate effects of treatment of RA on MA-mediated psycho-behaviors were studied by applying the one-sided trapezoidal tank diving test. The diving test of MA-treated zebrafish resulted that the dose of $25 \mathrm{mg} / \mathrm{l}$ of MA was anxiolytic compared to the test of saline-treated zebrafish as the control. But the diving pattern of the zebrafish exposed to RA as well as MA was novel and varied from both the control and MA-treated groups. The diving pattern was not similar to any of them and even the stress and speed of diving were less than the MA-treated group.

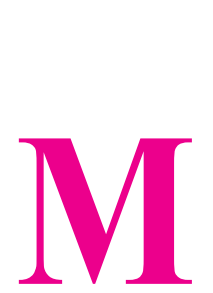

\section{Introduction}

ethamphetamine (MA) is a highly addictive dopaminergic stimulant (Kish, 2014), with severe neurotoxicity (Takeichi, Wang, \& Kitamura, 2012). The acute effects of MA include feelings of well-being, excitement, and heightened alertness (Freese, Miotto, \& Reback, 2002). These euphoric feelings result from altering the normal physiologic processing of several centrally-acting CNS monoamine neurotransmitters (Klasser \& Epstein, 2005).

MA can change some key biochemical mechanisms in the brain. It is associated with a variety of neurotoxic stress-mediated apoptosis. MA and its derivatives trigger oxidative stress in dopaminergic neurons and increase mitochondrial oxidant stress in terminals and dendrites through a Monoamine Oxidase (MAO)-dependent mechanism that results in the loss of Substantia Nigra pars compacta (SNc) dopaminergic neurons (Graves et al., 2017). MA noticeably increases dopamine release in the mesolimbic (dopamine) DA system, resulting in the formation of Reactive Oxygen Species (ROS) and oxidative damage (Jang et al., 2017), leading to programmed cell death. MA induces neuronal apoptosis through the Endoplasmic Reticulum (ER) stress signaling pathway (Jayanthi, Deng, Noailles, Ladenheim, $\&$ Cadet, 2004). Since MA acts as a powerful oxidant, it sounds a promising idea that using a potent antioxidant such as Rosmarinic Acid (RA) could counteract MA oxidative stress and its consequences completely or partially. RA ( $\alpha$-O-caffeoyl-3, 4-dihydroxyphenyl lactic acid) is an herbal phenolic. RA occurs in several taxa, including Lamiaceae and Boraginaceae families (Petersen, 1991). RA is known as an antioxidant with 
radical-scavenging (Erkan, Ayranci, \& Ayranci, 2008), anti-inflammatory (Kelm, Nair, Strasburg, \& DeWitt, 2000), astringent (Lee Xu, Kim, \& Park, 2008; Parnham \& Kesselring, 1985), antimutagen (Furtado et al., 2008), antibacterial (Chakraborty et al., 2007), antiviral property by reacting rapidly with viral coat proteins (Parnham \& Kesselring, 1985; Thiel et al., 1981), anticholinesterase (Orhan, Aslan, Kartal, Şener, \& Başer, 2008), antitumor (Furtado et al., 2008), hepatoprotective (Lima, Fernandes-Ferreira, \& Pereira-Wilson, 2006), and cardioprotective properties (Psotova, Chlopcikova, Miketova, \& Simanek, 2005).

Studying the oxidative stress-mediated by MA needs a biomarker. Since apoptosis is one of the consequences of extensive oxidative stress for the cell, we decided to evaluate the changes in the level of Casp3 mRNA. One of the critical elements in apoptosis is the activation of the caspases family. Inactive caspases, called procaspases, are present in all cells which are activated through a proteolytic process (Pop \& Salvesen, 2009) when it is needed. They are typically activated through two main ways: extrinsic and intrinsic pathways (Shalini, Dorstyn, Dawar, \& Kumar, 2015). Since both pathways activate procaspase-3 (Kumar, 2007), this protein can be an informative marker of apoptosis.

Deng et al. demonstrated that MA treatment causes cleavages of caspase- 3 proenzyme, which initiates $8 \mathrm{~h}$ post-MA exposure and is almost completely cleaved after $16 \mathrm{~h}$ (2002). In this research, we should know whether the MA effect on caspase- 3 is only restricted to the activation of the procaspase- 3 resource of the cell or it is more basic. In other words, we wanted to determine whether MA can influence the level of casp3a mRNA mainly due to its oxidative stress and, if it is so, whether $\mathrm{RA}$ as a powerful herbal antioxidant could counteract MA effects on the level of casp3a mRNA. Altering gene regulation mediated by MA has been demonstrated previously. Limanaqi, Gambardella, Biagioni, Busceti, and Fornai (2018) have attempted to include all the molecular procedures initiating at the pre-synaptic dopamine terminals to attain the nucleus of postsynaptic neurons related to the epigenetic events.

In rats, the percentages of rosmarinic acid present in the brain and the blood for $30 \mathrm{~min}$ after the administration was calculated as $23.7 \% \pm 2.4 \%$ and $0.40 \% \pm 0.02 \%$ of the administered amount, respectively. The brain/blood concentration fraction of the compounds reverberate their permeability to the brain, which could be restricted by the Blood-Brain Barriers (BBBs) (Falé, Madeira, Florêncio, Ascensão, \& Serralheiro, 2011), but MA concen- trations were highest at the first 2 min after administration in all tissues except the spleen (Rivière, Gentry, \& Owens, 2000). Obviously, the speed of these two agents to pass the BBB is different. We wonder whether the administration of a suitable nanoparticle can help increase the speed of RA penetration to CNS. Therefore, we used bionanocomposite of $\mathrm{ZnO} /$ chitosan and combined it with RA. In recent years, enormous hybrid materials on the base of chitosan have been developed, including oxide agents, metal nanoparticles, and conducting polymers (Li, Deng, Deng, Liu, \& Xin, 2010).

Chitosan is an abundant natural polysaccharide with numerous advantages like biodegradability, non-toxicity, biocompatibility, water permeability, and good mechanical strength. So it is appropriate for drug delivery (Youssef, El-Sayed, El-Sayed, Salama, \& Dufresne, 2016; Li et al., 2010; Youssef, El-Nahrawy, \& Hammad, 2017). The physicochemical properties of chitosan play a significant role in drug delivery (Bhattarai, Gunn, \& Zhang, 2010). Drugs targeting the CNS must cross the $\mathrm{BBB}$, which is the main obstacle for various molecules to enter the CNS tissue (Blasi, Giovagnoli, Schoubben, Ricci, \& Rossi, 2007), and chitosan enhances absorption across the epithelial membranes due to its mucoadhesive properties (Margret, Begum, \& Kumar, 2017). The existence of amino and hydroxyl groups in chitosan structure helps form covalent bonds with diverse materials like metals, DNA, and proteins (Youssef et al., 2017). Out of all other metal and semiconductor nanoparticles, $\mathrm{ZnO}$ nanoparticles are the most frequently studied and important one as they are used to synthesize biosensors, drug-delivery systems, biomedical developments, and agriculture (Bedi \& Kaur, 2015). Since chitosan has a great affinity to form metal complexes with zinc (Muzzarelli \& Sipos, 1971; Muzzarelli \& Tubertini 1969), the chitosan- $\mathrm{ZnO}$ complex attracted great interest for its potential to be used as UV protector and medicament (AbdElhady, 2012). But in this research, we used it as a drug career. Also, Yadollahi et al. (2015) investigated the $\mathrm{ZnO}$-chitosan bionanocomposite hydrogel beads as a drug delivery system.

We have designed two separate experiments in the present research work. First, we wanted to characterize the impact of a single dose function of the widely-used psychostimulant drug, MA, on the level of casp3a mRNA on the CNS. Second, we intended to determine anxiolytic or anxiogenic immediate effects of chronic exposure of $25 \mathrm{mg} / \mathrm{L}$ MA and assess the protective effects of RA on MA-mediated psychobehavior effects. To accomplish these two objectives, we needed an animal model, and we used zebrafish (Danio rerio). Zebrafish are widely 
used to study the molecular basis of neurobiology with applications in neurotoxicology and neuropharmacology (Linney, Upchurch, \& Donerly, 2004; Teraoka, Dong, \& Hiraga, 2003), especially addiction (Klee et al., 2012). Such studies in rodent models, including the analysis of gene-expression profiles and pharmacological manipulations, have provided a few clues to the molecular neuropathobiology of drug-induced neuroadaptation procedures (Wagner et al., 1980; Krasnova et al., 2008) due to the complexity of the addiction process. The presence of a good balance between simplicity and complexity of organs and systems makes zebrafish a much better substitute. For example, the fish nervous system is simpler than rodents but can still control various complex behaviors such as aggression, learning, addiction, locomotion, and so on (Ninkovic \& Bally-Cuif, 2006). Zebrafish has appeared a useful complementary model to study various neurobehavioral functions (Levin, Bencan, \& Cerutti, 2007). Anxiety-like behavior and stress responses in zebrafish have been shown through swimming patterns in novel aquarium tanks (Pietsaro, Kaslin, Anichtchik, \& Panula 2003; Levin et al., 2007). The developed test procedure took benefit of zebrafish natural tendency to swim at the bottom when introduced into a novel atmosphere and then gradually, over a few minutes, expands their swimming area to the higher regions of the tank (Levin et al., 2007). The orthologues of the casp3 gene in zebrafish are casp3a with 4 transcripts and casp3b with only one transcript. In this study, primers were designed for the casp3a gene.

\section{Methods}

\subsection{Animal and husbandry}

Adult zebrafish (5-7 months-old, males and females) were used in the experiments. They were provided by the Agricultural Biotechnology Research Institute of Iran, Gilan (ABRII). The fish were kept at approximately $28.5^{\circ} \mathrm{C}$ in a $14: 10$-h light/dark cycle. The housing and diving test tanks water was prepared by mixing distilled water and sea salts (Instant Ocean, $1.2 \mathrm{~g} / 20 \mathrm{~L}$ of H2O). Housing tanks for the fish were 3 -L containers. Fish were fed thrice daily with twice brine shrimp and once flake fish food.

\subsection{Chemicals and solution preparations}

Methamphetamine was legally provided by the research agency of the drug enforcement police. The gifted MA had the highest purity and was used in $25 \mathrm{mg} / \mathrm{L}$ concentration. The concentration of 40 and $30 \mathrm{mg} / \mathrm{L}$ was tried, but they made the zebrafish depressed and were not suitable for the diving test. Rosmarinic acid was purchased from Sigma Chemical Co. (St Louis, CA, USA). The $\mathrm{ZnO}$-chitosan nanoparticles were synthesized as per the standard chemical precipitation method (Abdelhady 2012). The experimental groups included control (saline), MA, MA+RA, MA+RA/NP, RA, and NP.

Saline (control) solution was made with $60 \mathrm{mg}$ sea salt dissolved into $0.5 \mathrm{~L}$ of distilled water.

Methamphetamine (MA) solution was prepared by $12.5 \mathrm{mg}$ of MA dissolved in $0.5 \mathrm{~L}$ of saline.

Rosmarinic Acid (RA) solution was prepared by $15 \mathrm{mg}$ of RA dissolved in $0.5 \mathrm{~L}$ of saline (antioxidant power of over $97 \%$ by DPPH test).

MA+RA solution was made by $12.5 \mathrm{mg}$ of MA and 15 $\mathrm{mg}$ of RA dissolved in $0.5 \mathrm{~L}$ of saline.

$\mathrm{MA}+\mathrm{RA} / \mathrm{NP}$ solution was prepared by $15 \mathrm{mg}$ of RA combined with ZnO-chitosan, according to SamzadehKermani and Miri (2015). Then it was dissolved in 0.5 L of MA solution.

Nanoparticle (NP) solution was prepared by the same amount of ZnO-chitosan used to prepare $\mathrm{RA}-\mathrm{ZnO}$-chitosan nanoparticle dissolved in $0.5 \mathrm{~L}$ of saline.

\subsection{Drug administration}

Each solution was administered by immersing 20 zebrafish into a drug-containing beaker $(500 \mathrm{~mL})$ for 20 minutes, once per day for 7 consecutive days. The drug exposure was performed between 9:00 and 11:00 AM. A time interval of 30 minutes was imposed between the end of the last chemical treatment and the start of either the molecular or the behavioral examination.

\subsection{Quantitative Reverse Transcription-Poly- merase Chain Reaction (qRT-PCR)}

The zebrafish were sacrificed by the ice water shock $30 \mathrm{~min}$ after the last treatment. The brains from the adult fish were dissected and grouped in 3 samples ( 5 brains in each). Total RNA was extracted for each sample according to the Trizol RNA extraction protocol (RNA Isolation Protocol 2018) with slight modifications. RNA integrity and quantification were assessed to be in the desired range by gel electrophoresis and 260/280 nm OD ratio. The total extracted RNA was reverse-transcribed to the first-strand cDNA and amplified using HyperscriptTM one-step RT-PCR mastermix, HyperscriptTM Reverse Transcriptase, and custom primers. Primers of casp3a 


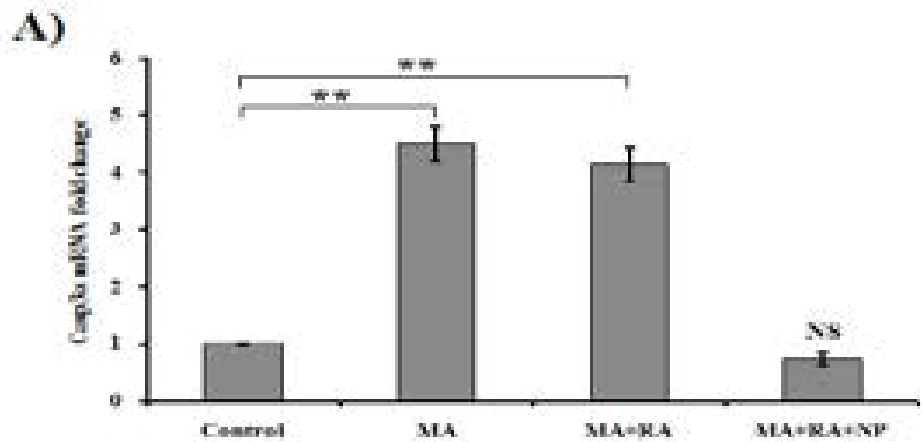

B)

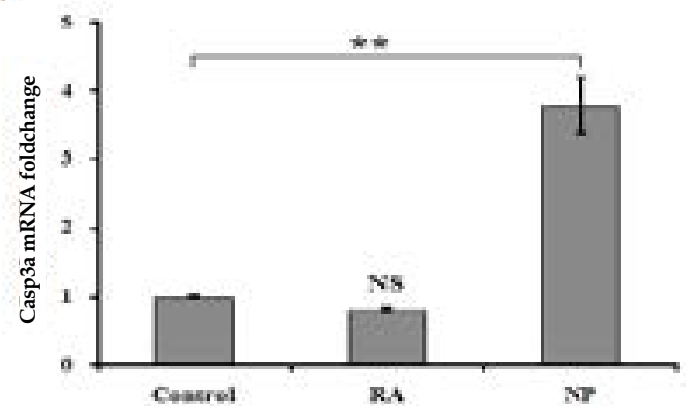

NEUR JSCIENCE

Figure 1. Changes in the casp3a mRNA level in the brain samples of fish after different treatments condition

Relative casp3a mRNA expression was calculated using the $2^{-\Delta \Delta \mathrm{ct}}$ method by quantitative reverse transcription-polymerase chain reaction (qRT-PCR), using gadph mRNA level as reference. The saline-treated group was served as the internal control.

MA: Methamphetamine; RA: Rosmarinic acid; NP: ZnO-chitosan nanoparticle. A: The qRT-PCR is analyzed after single exposure per day for seven consecutive days results in elevated casp3a mRNA expression (" $\mathrm{P}<0.01$, by 1-way ANOVA) in the MA group. Pre-treatment of fish with NP-coated RA reduced the casp3a expression as the control group; B. Significant changes in mRNA levels of casp3a were not observed in the RA animal group. While NP treatment has remarkable effect on casp3a expression ("P<0.01, by 1-way ANOVA).

were designed manually, and the sequences of them were as follows: forward, 5' TCGGTTCTCGCTGTTGAAGG 3' and reverse, 5' GTCTCCGTATCCGCATGTCC3'. Sequences of gapdh primers were as follows (McCurley \& Callard, 2008): forward, 5' GTGGAGTCTACTGGTGTCTTC 3' and reverse, 5' GTGCAGGAGGCATTGCTTACA 3'. The qRT-PCR was performed using QIAGEN One-Step RT-PCR Kit. Thirty-five thermal cycles were carried out as follows: $95^{\circ} \mathrm{C}$ for $30 \mathrm{~s}, 59^{\circ} \mathrm{C}$ for $30 \mathrm{~s}$, and $72^{\circ} \mathrm{C}$ for $60 \mathrm{~s}$. The final incubation was set at $72^{\circ} \mathrm{C}$ for $10 \mathrm{~min}$. The quantification was made applying 2 (-delta delta $\mathrm{Ct}$ ) calculations.

\subsection{One-sided trapezoidal tank diving test}

There were three different treatment groups of fish: saline, MA, MA pretreated by RA. Since it is previously revealed and also demonstrated in this research, $\mathrm{ZnO}$ induces oxidative stress. To prevent the probable effects of $\mathrm{ZnO}$ on animal's brains and behavior, MA and RA$\mathrm{ZnO}$-chitosan groups were replaced with MA pretreated by the RA group. Pretreatment of RA was performed 30 min before MA. Lee et al. (2008) also treated RA 30 min before the induction of pro-oxidant challenge.

The diving patterns of 3 zebrafish from each group were traced one by one and compared to each other. Zebrafish were placed individually in a $1.5-\mathrm{L}$ trapezoidal tank $(16 \mathrm{~cm}$ height $\times 27.5 \mathrm{~cm}$ top $\times 23.5 \mathrm{~cm}$ bottom $\times 7.1$ cm width) filled with $1350 \mathrm{~mL}$ of home tank water. Tanks were put on a stable surface and were divided into three equal virtual horizontal parts. Once the fish is relocated into one-sided trapezoidal tanks, a side view camera recorded the swimming behavior over a 5-min period. The following endpoints were recorded: time spent in each part of the tank(s) and total traveled distance. The choice of position (bottom vs. upper levels) was considered the anxiety index. Choice of dwelling on the bottom was near a position of safety.

\subsection{Statistical analysis}

The obtained data were presented as mean values and analyzed by t-test, 1-way Analysis of Variance (ANO- 

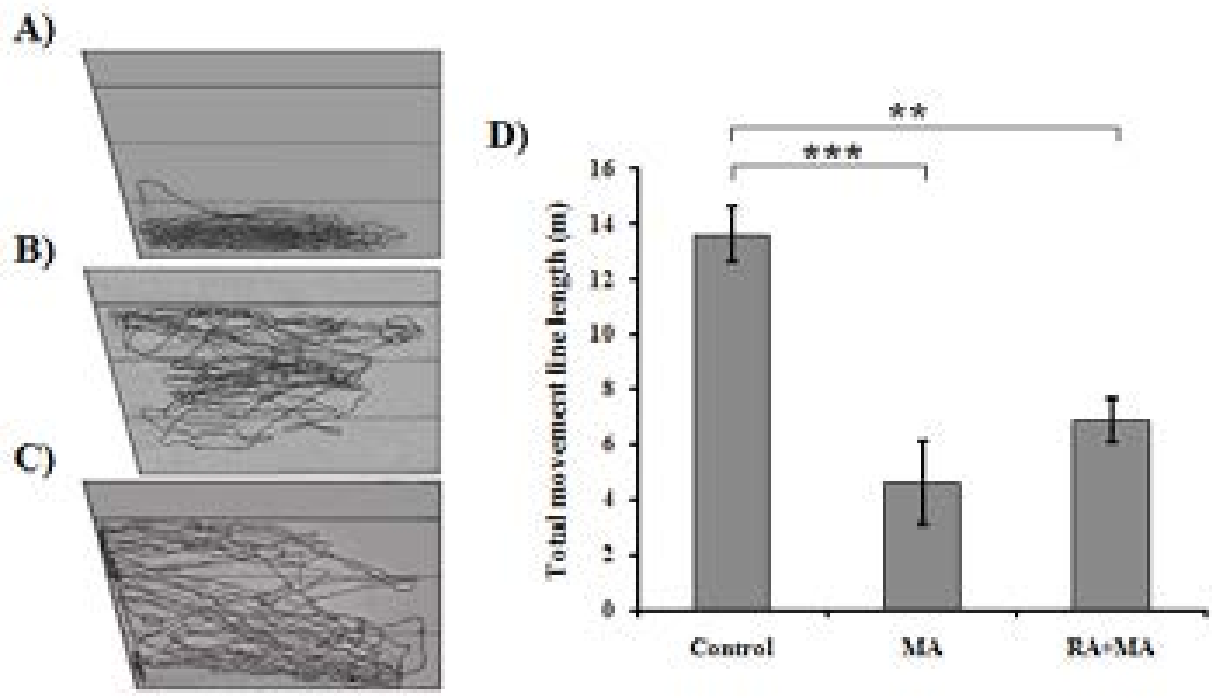

Figure 2. Effect of MA and RA on dwelling and swimming patterns of fish

Movement traces of cohorts of three distinct treated fish were drawn based on observation of one-sided trapezoidal tank diving test: A: Control; B: MA; and C: MA pretreated by RA. The traces of fish movements indicate remarkable changes in their dwelling and swimming pattern compared to the control ones. The control animals traveled for a total distance of $13.6 \mathrm{~m}$ and stayed at the bottom of the tank. MA administration has changed the fish behavior in a way that traveled for $4.64 \mathrm{~m}$ and continuous vertical shift between top and middle of the test tank. Pre-treatment of fish by RA reduced the negative effects of MA. They resembled the swimming pattern of control fish up to some extent. The overall dwelling and swimming patterns of each treatment group's fish were found to be significantly different ${ }^{* *} \mathrm{P}<0.01$, by 2 -way ANOVA).

VA), and 2-way ANOVA followed by Dunnett post-hoc comparisons using SPAW (predictive analytics software) version 19.0. The $P$ value of 0.05 was considered as the minimum level of significance.

\section{Results}

3.1. qRT-PCR Data: Changes in Casp3a mRNA Level in the brain

qRT-PCR results (Figure 1A) demonstrated that increases in the casp3a mRNA levels were up to 18-fold for MA, 16.8-fold for MA cotreated with RA, and 14-fold for the $\mathrm{ZnO}$-chitosan-treated fish group. MA cotreated with $\mathrm{RA}-\mathrm{ZnO}$-chitosan brought back the casp3a mRNA level near to that of the control group (2.8-fold), unlike the co-treatment of MA and RA that RA was insufficient to reduce the increased mRNA level. RA alone caused a little reduction in casp3a mRNA level as compared to the control group (Figure 1B) $(\mathrm{n}=3, \mathrm{P}<0.01)$.

\subsection{Behavioral Test: One-Sided Trapezoidal Tank Diving Test}

Behavioral assessment of all groups (cohorts) and total distance traveled in 5 minutes was performed in three replicates, but one for each group is shown. It has been reported that $\mathrm{ZnO}$ induces oxidative stress. Thus, to prevent the probable effects of $\mathrm{ZnO}$ on animal's brain and behavior, the MA and RA-ZnO-chitosan groups were replaced with MA pretreated by the RA group. Pretreatment of RA was performed $30 \mathrm{~min}$ before MA treatment.

All fish from the MA group started dwelling in the middle of the tank. The zebrafish that its behavior is shown in Figure $2 \mathrm{~B}$ spent about $42.66 \%$ of the time in the middle. They spent less than half of the total time in the top part $(48.66 \%)$ and the rest of the time in the bottom (Figure 3 ). The fish had 3 transitions to the bottom for the sake of curiosity but did not spend much time there and came back soon to the upper portions (data not shown). MA group swimming exploration was stress-free and easy.

All fish from the MA pretreated by RA group started dwelling in the top part of the tank, the furthest area from the position of safety, but they spend most of the time at the bottom. The zebrafish swimming pattern shown in Figure $2 \mathrm{C}$ spent $32 \%$ of the total time on top, more than $29.33 \%$ in the middle, and the rest of the time (38.67\%) at the bottom (Figure 3). The zebrafish had more than 10 transitions to the bottom and special attention to the trapezoidal side. 


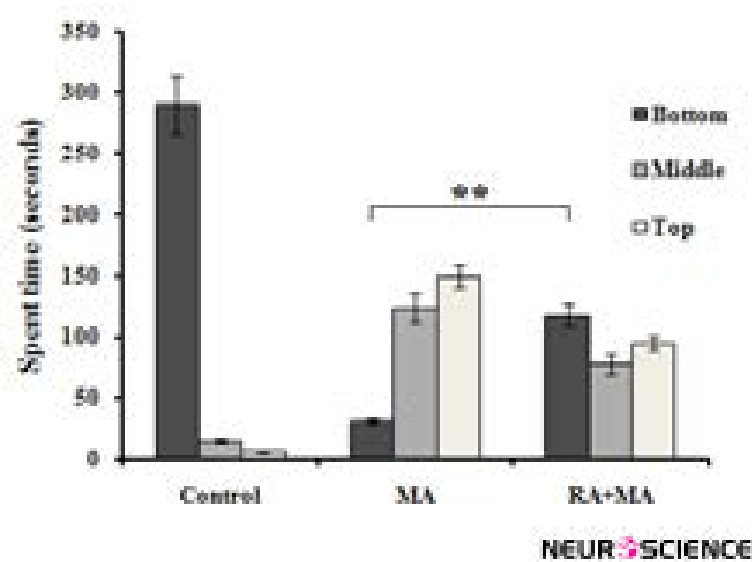

Figure 3. The total time spent by each individual fish from different treatments in the bottom, middle, and top of the one-sided trapezoidal tank

The control group (saline-treated) displays unique features of a stressed fish, which stayed in the bottom of the tank for about 290 seconds, whereas MA exposed fish spent mostly in the top of the tank (145 seconds). The pretreated RA group exposed to MA renders the MA effect and makes the fish stay for a longer time (115 seconds) in the bottom of the aquarium. The treatments caused significant different behavioral responses to the new environment, evident by noticeable stay-time at the bottom or top of the test tank $\left({ }^{* *} \mathrm{P}<0.01\right.$, by 1 -way ANOVA).

All the control fish spent almost a whole $5 \mathrm{~min}$ at the bottom, showing stressful back and forth movements (Figure 2A). Generally, the control fish had one or two transitions (near the trapezoidal side) to the middle part but, it sounds that the trapezoidal side was unpleasant to them, so they came back to the bottom soon.

The total distance $(\mathrm{m})$ traveled in 5 minutes by all three treated zebrafish is shown in Figure 2D. The total distance traveled was $6.87 \mathrm{~m}$ for the MA pretreated by $\mathrm{RA}, 4.64 \mathrm{~m}$ for MA, and $13.6 \mathrm{~m}$ for control fish groups.

\section{Discussion}

4.1. Methamphetamine and Casp3a mRNA Level increase in the Brain of Zebrafish and RA-ZnOchitosan Counteracting it Remarkably

Increasing the casp3a mRNA levels up to 18 -fold by MA shows that MA can up-regulate the casp3a gene expression. Producing indelible genetic modifications shifting neuronal phenotype and induce behavioral alterations by MA (Limanaqi et al., 2018) and decreasing cleaved caspase- 3 expression by RA (Zhang et al., 2017) have been demonstrated before by lots of research works. This paper aimed to demonstrate the potential of rosmarinic acid to counteract the deleterious effects of MA-mediated oxidative stress due to its high antioxidant power.

Cotreatment of MA with RA increases casp3a mRNA level up to 16.8-fold, so near to the level of the MAtreated group. This result may indicate that RA cannot counteract the effects of MA-mediated oxidative stress at first sight. However, co-treatment of MA with RA$\mathrm{ZnO}$-chitosan decreased the casp3a mRNA level down to 2.8-fold compared to the level of the MA-treated group. It obviously shows that inefficient permeability of RA compared to MA through the BBB makes RA unable to function punctually. Treatment by $\mathrm{ZnO}$-chitosan increases casp3a mRNA level up to 14-fold.

$\mathrm{ZnO}$ nanoparticles can induce the excess generation of Reactive Oxygen Species (ROS), leading to cell demise (Xia et al., 2006; Ryter et al., 2007; Long et al., 2006; Lovric et al., 2005; Lewinski, Colvin, Drezek, 2008). The cytotoxic effect of $\mathrm{ZnO}$ nanoparticles on activity and transcription levels of the antioxidant enzyme has been illustrated in co-cultured $\mathrm{C} 2 \mathrm{C} 12$ and 3T3-L1 cells (Pandurangan \& Kim, 2015). Moreover, the dose-dependent deleterious effects of $\mathrm{ZnO}$ nanoparticles on antioxidant enzyme activity in adipocytes have been reported (Muthuraman, Ramkumar, \& Kim, 2014).

Pandurangan and Kim (2015) reported that the induction of oxidative stress is a vital part of the cytotoxicity of $\mathrm{ZnO}$ nanoparticles. We also observed such an effect by increased casp3a mRNA level in ZnO-chitosan-treated zebrafish, which was about $78 \%$ of what was observed in the MA-treated group. These results indicate two points; first, the weak deficiency of RA to inhibit the augmentation of casp3a mRNA (in the co-treatment of MA with $\mathrm{RA}$ ) is due to its deficiency in crossing the blood-brain barrier as fast as MA, not the deficiency in the antioxidant power of RA. Second, RA and ZnO-chitosan can eliminate each other's downsides. $\mathrm{ZnO}$ assists RA to cross the $\mathrm{BBB}$, and RA is such a powerful antioxidant that it is not only able to counteract MA-mediated oxidative stress but is almost able to counteract $\mathrm{ZnO}$-mediated oxidative stress, too. It seems that the antioxidant power of RA has yet been underestimated. RA and its derivatives present plentifully in Salvia species (Lu \& Foo, 1999). A balanced diet affords antioxidant agents and prevents the disease condition by delaying the biological oxidative processes to contribute to aging and disease risk. There is a well-established connection between the increased use of antioxidants and decreased cancer occurrence (Seifried, McDonald, Anderson, Greenwald, \& Milner, 2003). That is why antioxidant supplements are regularly recommended as ingredients of a cancer- 
preventing diet (Ames, 1999; Prasad, Kumar, Kochupillai, \& Cole, 1999). However, the production of excess levels of ROS are essential for the commencement of internal cell programs towards cell suicide (apoptosis) that are vital protective mechanisms killing cancerous cells (Weijl, Cleton, Osanto, 1997; Kuipers \& Lafleur, 1998).

$\mathrm{ZnO}$ is an inorganic material with a wide range of promising applications (Neumark et al., 2007), but inducing high oxidative stress is not a feature to be ignored. Fortunately, our $\mathrm{ZnO}$-based nanoparticle was combined with a powerful antioxidant. Whether combining $\mathrm{ZnO}$ with an antioxidant is enough to counteract all the side effects of $\mathrm{ZnO}$ is a subject that needs more research work.

The main challenge that we had was the primer dimers in the samples with a low amount of casp3a mRNA. In quantitative real-time PCR, the total amount of DNA is measured after each PCR cycle by fluorescent signals produced directly in relation to the number of PCR products (amplicons) generated. Data are collected at the exponential phase of the reaction. In the present study, fluorescent reporters used in real-time PCR included double-stranded DNA (dsDNA)-binding dyes that hybridize with PCR product during amplification. By plotting fluorescence intensity against the cycle number, an amplification plot will be generated, representing the accumulation of amplicon throughout the entire PCR reaction. Binding the dye to the primer dimers increases the product's accumulation in three saline, RA, and MA co-treated with $\mathrm{RA}-\mathrm{ZnO}$-chitosan samples. In real-time PCR, the amount of primers must be equal in all samples. When there is a noticeable gap in the amount of template mRNA in some samples, primer dimers would have been seen in samples with a low level of the desired mRNA.

\subsection{The Anxiolytic Effect of Methamphetamine (25 $\mathrm{mg} / \mathrm{L}$ ) on the Zebrafish}

Unlike the level of casp3a mRNA wherein treatment by $\mathrm{RA} / \mathrm{ZnO} /$ chitosan brought it back near to the level of the saline group, the swimming behavior of MA pretreated by RA group was unique. The observed behavior was neither similar to MA nor saline. Although the fish from the MA pretreated by RA group were calmer and less stressed than the MA-treated group and started dwelling in the top, generally they spent about $70 \%$ of the time at the middle and bottom of the tank, but the MA group spent about $90 \%$ of the time at the middle and top of the tank. The total distance traveled in 5 minutes of MA was the least of all the cases, which means this group's swimming speed was slower than that of the RA+MA group. The swimming and muscle movement of both groups were just normal, but the MA group was more relaxed. It has been reported previously that RA treatment for 7 or 14 days produces an antidepressant-like effect in mice (Ito et al., 2008), and extract of lemon balm, which is RA rich, inhibits GABA transaminase, enhances GABAergic activity in the brain, and provides a calming effect on depression and also in anxiety (Dubey, Sahu, Kumari, Yadav, \& Sahu, 2018). Our findings revealed that $25 \mathrm{mg} / \mathrm{L}$ dose of MA is anxiolytic for zebrafish, and RA has complex effects on MA-mediated psychobehavior. Although the precise mechanisms underlying the protective potential of RA are not entirely understood, the putative oxidative and enhancing GABAergic activity of RA might explain its effect on MA-mediated psychobehavior.

The molecular mechanisms involved in these processes should be studied in the future, but this study proved that RA combined with a suitable nanoparticle could significantly counteract MA-mediated oxidative stress and some of the consequences. However, it does not reduce the anxiolytic effects of MA and even enhances it. Since MA and MA hydrochloride hold allure because their immediate effects produce feelings of well-being, an auxiliary drug that diminishes the mentioned feelings has no potential marketability. In other words, drug users do not like to consume an auxiliary drug that is not accordant with the incentive of the drug of abuse.

Based on our study, RA merits paying attention to and allocate more funds to discover the molecular basis of its action against MA-mediated oxidative stress. Moreover, it is noteworthy to produce medicines based on RAnanoparticle combinations that eliminate the side effects and increase drug delivery and efficiency.

\section{Ethical Considerations}

\section{Compliance with ethical guidelines}

This study was approved by the Ethics Committee of the University of Zabol.

\section{Funding}

This research project was supported by the University of Zabol (Grant No. UOZ-GR-9517-51) Zabol City, Iran.

\section{Authors' contributions}

Data collection: Vida Nikshenas Shahrestani; Investigation and editing: Mohammad Haddadi; Nanoparticle synthesis and administration to our model organism: Ali Reza Samzadeh Kermani. 


\section{Conflict of interest}

The authors declared no conflict of interest.

\section{Acknowledgments}

The authors would like to thank the Research and Technology Chancellor, the University of Tehran, for their support and the facilities. We thank Dr. Jamshidi (Agricultural Biotechnology Research Institute of Iran, Gilan) for her contribution to molecular analysis on qRT-PCR results and for providing the zebrafish. We also thank $\mathrm{Mr}$. Faramarz Dahmardeh for his close cooperation with Ms. Nickshenas in aquarium preparation and maintenance.

\section{References}

AbdElhady, M. M. (2012). Preparation and characterization of chitosan/zinc oxide nanoparticles for imparting antimicrobial and UV protection to cotton fabric. International Journal of Carbohydrate Chemistry, 2012. [DOI:10.1155/2012/840591]

Ames, B. N. (1999). Micronutrient deficiencies: A major cause of DNA damage. Annals of the New York Academy of Sciences, 889(1), 87-106. [DOI:10.1111/j.1749-6632.1999.tb08727.x] [PMID]

Bedi, P., \& Kaur, A. (2015). An overview on uses of zinc oxide nanoparticles. World Journal of Pharmacy and Pharmaceutical Sciences, 4(12), 1177-96. https://www.researchgate.net/ publication/338819464_AN_OVERVIEW_ON_USES_OF_ ZINC_OXIDE_NANOPARTICLES

Bhattarai, N., Gunn, J., \& Zhang, M. (2010). Chitosan-based hydrogels for controlled, localized drug delivery. Advanced Drug Delivery Reviews, 62(1), 83-99. [DOI:10.1016/j. addr.2009.07.019] [PMID]

Blasi, P., Giovagnoli, S., Schoubben, A., Ricci, M., \& Rossi, C. (2007). Solid lipid nanoparticles for targeted brain drug delivery. Advanced Drug Delivery Reviews, 59(6), 454-77. [DOI:10.1016/j.addr.2007.04.011] [PMID]

Chakraborty, D. M. S., Chakraborty, J., Bhattacharyaa, P. K., Bandyopadhyay, A., Mitra, A., \& Gupta, K. (2007). Antimicrobial activity of leaf extract of Basilicum polystachyon (L) Moench. Indian Journal of Experimental Biology, 45, 744-8. [PMID]

Deng, X., Cai, N. S., McCoy, M. T., Chen, W., Trush, M.A. \& Cadet, J. L. (2002). Methamphetamine induces apoptosis in an immortalized rat striatal cell line by activating the mitochondrial cell death pathway. Neuropharmacology, 42(6), 837-45. [DOI:10.1016/S0028-3908(02)00034-5]

Dubey, T., Sahu, G., Kumari, S., Yadav, B.S. \& Sahu, A.N. (2018). Role of herbal drugs on neurotransmitters for treating various CNS disorders: A review. Indian Journal of Traditional Knowledge, 17(1), 113-21. http://nopr.niscair.res.in/ handle/123456789/43142

Erkan, N., Ayranci, G., \& Ayranci, E. (2008). Antioxidant activities of rosemary (Rosmarinus Officinalis L.) extract, blackseed
(Nigella sativa L.) essential oil, carnosic acid, rosmarinic acid and sesamol. Food Chemistry, 110(1), 76-82. [DOI:10.1016/j. foodchem.2008.01.058] [PMID]

Falé, P. L., Madeira, P. J. A., Florêncio, M. H., Ascensão, L., \& Serralheiro, M. L. M. (2011). Function of Plectranthus barbatus herbal tea as neuronal acetylcholinesterase inhibitor. Food \& Function, 2(2), 130-6. [DOI:10.1039/C0FO00070A] [PMID]

Freese, T. E., Miotto, K., \& Reback, C. J. (2002). The effects and consequences of selected club drugs. Journal of Substance Abuse Treatment, 23(2), 151-6. [DOI:10.1016/S0740-5472(02)00267-2]

Furtado, M. A., de Almeida, L. C. F., Furtado, R. A., Cunha, W R., \& Tavares, D. C. (2008). Antimutagenicity of rosmarinic acid in Swiss mice evaluated by the micronucleus assay. Mutation Research/Genetic Toxicology and Environmental Mutagenesis, 657(2), 150-4. [DOI:10.1016/j.mrgentox.2008.09.003] [PMID]

Graves, S. M., Xie, Z., Zampese, E., Stout, K. A., Tai, R. A., \& Schwarzschild, S. E., et al. (2017). Methamphetamine-induced mitochondrial oxidant stress mediated by monoamine oxidase metabolism of dopamine. The FASEB Journal, 31(S1), 987-3. [DOI:10.1096/fasebj.31.1_supplement.987.3]

Ito, N., Yabe, T., Gamo, Y., Nagai, T., Oikawa, T., \& Yamada, H., et al. (2008). Rosmarinic acid from Perillae Herba produces an antidepressant-like effect in mice through cell proliferation in the hippocampus. Biological and Pharmaceutical Bulletin, 31(7), 1376-80. [DOI:10.1248/bpb.31.1376] [PMID]

Jang, E. Y., Yang, C. H., Hedges, D. M., Kim, S. P., Lee, J. Y., \& Ekins, T. G., et al. (2017). The role of reactive oxygen species in methamphetamine self-administration and dopamine release in the nucleus accumbens. Addiction Biology, 22(5), 1304-15. [DOI:10.1111/adb.12419] [PMID] [PMCID]

Jayanthi, S., Deng, X., Noailles, P. A. H., Ladenheim, B., \& Cadet, J. L. (2004). Methamphetamine induces neuronal apoptosis via cross-talks between the endoplasmic reticulum and mitochondria-dependent death cascades. The FASEB Journal, 18(2), 238-251. [DOI:10.1096/fj.03-0295com] [PMID]

Kelm, M. A., Nair, M. G., Strasburg, G. M., \& DeWitt, D. L. (2000). Antioxidant and cyclooxygenase inhibitory phenolic compounds from Ocimum sanctum Linn. Phytomedicine, 7(1), 7-13. [DOI:10.1016/S0944-7113(00)80015-X]

Kish, S. J. (2014). The pathology of methamphetamine use in the human brain. In M. Kuhar (Ed.), The Effects of Drug Abuse on the Human Nervous System (pp. 203-297). Amsterdam: Elsevier Science. [DOI:10.1016/ B978-0-12-418679-8.00008-3]

Klasser, G. D., \& Epstein, J. (2005). Methamphetamine and its impact on dental care. Journal of the Canadian Dental Association, 71(10). https://www.cda-adc.ca/jcda/vol-71/issue-10/759.pdf

Klee, E. W., Schneider, H., Clark, K. J., Cousin, M. A., Ebbert, J. O., \& Hooten, W. M., et al. (2012). Zebrafish: A model for the study of addiction genetics. Human Genetics, 131(6), 977-1008. [DOI:10.1007/s00439-011-1128-0] [PMID] [PMCID]

Krasnova, I. N., Li, S. M., Wood, W. H., McCoy, M. T., Prabhu, V. V., \& Becker, K. G., et al. (2008). Transcriptional responses to reinforcing effects of cocaine in the rat hippocampus and cortex. Genes, Brain, and Behavior, 7(2), 193-202. [DOI:10.1111/ j.1601-183X.2007.00338.x] [PMID]

Kuipers G.K., \& Lafleur, M.V.M. (1998). Characterization of DNA damage induced by gamma-radiation derived water radicals, 
using DNA repair enzymes. International Journal of Radiation Biology, 74(4), 511-9. [DOI: 10.1080/095530098141384] [PMID]

Kumar, S. (2007). Caspase function in programmed cell death. Cell Death \& Differentiation, 14(1), 32-43. [DOI:10.1038/ sj.cdd.4402060] [PMID]

Lee, H. J., Cho, H. S., Park, E., Kim, S., Lee, S. Y., Kim, C. S., ... \& Chun, H. S. (2008). Rosmarinic acid protects human dopaminergic neuronal cells against hydrogen peroxide-induced apoptosis. Toxicology, 250(2-3), 109-15. [DOI:10.1016/j. tox.2008.06.010] [PMID]

Lee, S.Y., Xu, H., Kim, Y. K., \& Park, S.U. (2008). Rosmarinic acid production in hairy root cultures of Agastache rugosa Kuntze. World Journal of Microbiology \& Biotechnology, 24, 969-72. [DOI:10.1007/s11274-007-9560-y]

Levin, E. D., Bencan, Z., \& Cerutti, D.T. (2007). Anxiolytic effects of nicotine in zebrafish. Physiology \& Behavior, 90(1), 54-8. [DOI:10.1016/j.physbeh.2006.08.026] [PMID]

Lewinski, N., Colvin, V., \& Drezek, R. (2008). Cytotoxicity of nanoparticles. Small, 4(1), 26-49. [DOI:10.1002/smll.200700595] [PMID]

Li, L. H., Deng, J. C., Deng, H. R., Liu, Z. L., \& Xin, L. (2010). Synthesis and characterization of chitosan/ZnO nanoparticle composite membranes. Carbohydrate Research, 345(8), 994-8. [DOI:10.1016/j.carres.2010.03.019] [PMID]

Lima, C. F., Fernandes-Ferreira, M., \& Pereira-Wilson, C. (2006) Phenolic compounds protect HepG2 cells from oxidative damage: relevance of glutathione levels. Life Sciences, 79(21) 2056-68. [DOI:10.1016/j.lfs.2006.06.042] [PMID]

Limanaqi, F., Gambardella, S., Biagioni, F., Busceti, C. L. \& Fornai, F. (2018). Epigenetic effects induced by methamphetamine and methamphetamine-dependent oxidative stress. Oxidative Medicine and Cellular Longevity, 2018. [DOI:10.1155/2018/4982453] [PMID] [PMCID]

Linney, E., Upchurch, L., \& Donerly, S. (2004). Zebrafish as a neurotoxicological model. Neurotoxicology and Teratology, 26(6), 709-18. [DOI:10.1016/j.ntt.2004.06.015] [PMID]

Long, T. C., Saleh, N., Tilton, R. D., Lowry, G. V., \& Veronesi, B. (2006). Titanium dioxide (P25) produces reactive oxygen species in immortalized brain microglia (BV2): Implications for nanoparticle neurotoxicity. Environmental Science \& Technology, 40(14), 4346-52. [DOI:10.1021/es060589n] [PMID]

Lovrić, J., Cho, S. J., Winnik, F. M., \& Maysinger, D. (2005). Unmodified cadmium telluride quantum dots induce reactive oxygen species formation leading to multiple organelle damage and cell death. Chemistry \& Biology, 12(11), 1227-34. [DOI:10.1016/j.chembiol.2005.09.008] [PMID]

Lu, Y., \& Foo, L. Y. (1999). Rosmarinic acid derivatives from Salvia officinalis. Phytochemistry, 51, 91-4. [DOI:10.1016/S00319422(98)00730-4]

Margret, A. A., Begum, T. N., \& Kumar, A. G. (2017). Discerning the potential of chitosan extracted from bioresources as a competent brain drug carrier to cross the blood brain barrier. Indian Journal of Pharmaceutical Sciences, 79(2), 186-96. [DOI:10.4172/pharmaceutical-sciences.1000216]

McCurley, A. T., \& Callard, G. V. (2008). Characterization of housekeeping genes in zebrafish: Male-female differences and effects of tissue type, developmental stage and chemical treat ment. BMC Molecular Biology, 9(1), 1-12. [DOI:10.1186/14712199-9-102] [PMID] [PMCID]

Muthuraman, P., Ramkumar, K., \& Kim, D. H. (2014). Analysis of dose-dependent effect of zinc oxide nanoparticles on the oxidative stress and antioxidant enzyme activity in adipocytes. Applied Biochemistry and Biotechnology, 174(8), 2851-63. [DOI:10.1007/s12010-014-1231-5] [PMID]

Muzzarelli, R. A. \& Sipos, L. (1971). Chitosan for the collection from seawater of naturally occurring zinc, cadmium lead and copper. Talanta, 18(9), 853-8. [DOI:10.1016/00399140(71)80141-8] [PMID]

Muzzarelli, R. A. \& Tubertini, O. (1969). Chitin and chitosan as chromatographic supports and adsorbents for collection of metal ions from organic and aqueous solutions and sea-water. Talanta, 16(12), 1571-7. [DOI:10.1016/0039-9140(69)80218-3] [PMID]

Neumark, G., Gong, Y., \& Kuskovsky, I. (2007). Doping aspects of Zn-based wide-band-gap semiconductors. Springer Handbook of Electronic and Photonic Materials, 843. [DOI:10.1007/9780-387-29185-7_35]

Ninkovic, J., \& Bally-Cuif, L. (2006). The zebrafish as a model system for assessing the reinforcing properties of drugs of abuse Methods, 39(3), 262-74. [DOI:10.1016/j.ymeth.2005.12.007] [PMID]

Orhan, I., Aslan, S., Kartal, M., Şener, B., \& Başer, K. H. C. (2008). Inhibitory effect of Turkish Rosmarinus officinalis L. on acetylcholinesterase and butyrylcholinesterase enzymes. Food Chemistry, 108(2), 663-8. [DOI:10.1016/j.foodchem.2007.11.023] [PMID]

Pandurangan, M., \& Kim, D. H. (2015). In vitro toxicity of zinc oxide nanoparticles: A review. Journal of Nanoparticle Research, 17(3), 158. [DOI:10.1007/s11051-015-2958-9]

Parnham, M. J., \& Kesselring, K. (1985). Rosmarinic acid. Drugs of the Future, 10, 756-7. [DOI:10.1358/dof.1985.010.09.71743]

Petersen, M. S. (1991). Characterization of rosmarinic acid synthase from cell cultures of Coleus blumei. Phytochemistry, 30(9), 2877-81. [DOI:10.1016/S0031-9422(00)98217-7]

Pietsaro, N., Kaslin, J., Anichtchik, O.V., \& Panula, P. (2003) Modulation of the histaminergic system and behavior by afluoromethylhistidine in zebrafish. Journal of Neurochemistry, 86(2), 432-41. [DOI:10.1046/j.1471-4159.2003.01850.x] [PMID]

Pop, C., \& Salvesen, G. S. (2009). Human caspases: Activation, specificity, and regulation. Journal of Biological Chemistry, 284(33) 21777-81. [DOI:10.1074/jbc.R800084200] [PMID] [PMCID]

Prasad, K. N., Kumar, A., Kochupillai, V., \& Cole, W. C. (1999). High doses of multiple antioxidant vitamins: essential ingredients in improving the efficacy of standard cancer therapy. Journal of the American College of Nutrition, 18(1), 13-25. [DOI:1 0.1080/07315724.1999.10718822] [PMID]

Psotova, J., Chlopcikova, S., Miketova, P., \& Simanek, V. (2005) Cytoprotectivity of Prunella vulgaris on doxorubicin treated rat cardiomyocytes. Fitoterapia, 76, 556-61. [DOI:10.1016/j.fitote.2005.04.019] [PMID]

Ryter, S. W., Kim, H. P., Hoetzel, A., Park, J. W., Nakahira, K. Wang, X., \& Choi, A. M. (2007). Mechanisms of cell death in oxidative stress. Antioxidants \& Redox Signaling, 9(1), 49-89. [DOI:10.1089/ars.2007.9.49] [PMID] 
Rivière, G. J., Gentry, W. B., \& Owens, S. M. (2000). Disposition of methamphetamine and its metabolite amphetamine in brain and other tissues in rats after intravenous administration. Journal of Pharmacology and Experimental Therapeutics, 292(3), 10427. https://jpet.aspetjournals.org/content/292/3/1042.short

Samzadeh-Kermani, A., \& Miri, S. (2015). Synthesis, characterization and bactericidal property of chitosan-graft-polyaniline/montmorillonite/ZnO nanocomposite. Korean Journal of Chemical Engineering, 32(6), 1137-41. [DOI:10.1007/s11814-0140285-y]

Seifried, H. E., McDonald, S. S., Anderson, D. E., Greenwald, P., \& Milner, J. A. (2003). The antioxidant conundrum in cancer. Cancer Research, 63(15), 4295-8. https://cancerres.aacrjournals.org/content/63/15/4295.short

Shalini, S., Dorstyn, L., Dawar, S. \& Kumar, S. (2015). Old, new and emerging functions of caspases. Cell Death and Differentiation, 22(4), 526-39. [DOI:10.1038/cdd.2014.216] [PMID] [PMCID]

Takeichi, T., Wang, E. L., \& Kitamura, O. (2012). The effects of low-dose methamphetamine pretreatment on endoplasmic reticulum stress and methamphetamine neurotoxicity in the rat midbrain. Legal Medicine, 14(2), 69-77. [DOI:10.1016/j.legalmed.2011.12.004] [PMID]

Teraoka, H., Dong, W., \& Hiraga, T. (2003). Zebrafish as a novel experimental model for developmental toxicology. Congenital Anomalies, 43(2), 123-32. [DOI:10.1111/j.1741-4520.2003. tb01036.x] [PMID]

Thiel, K. D., Helbig, B., Klöcking, R., Wutzler, P., Sprössig, M., \& Schweizer, H. (1981). Comparison of the in vitro activities of ammonium humate and of enzymically oxidized chlorogenic and caffeic acids against type 1 and type 2 human herpes virus (author's transl). Die Pharmazie, 36(1), 50-3. [PMID]

Wagner, G. C., Ricaurte, G. A., Seiden, L. S., Schuster, C. R., Miller, R. J., \& Westley, J. (1980). Long-lasting depletions of striatal dopamine and loss of dopamine uptake sites following repeated administration of methamphetamine. Brain Research, 181(1), 151-60. [DOI:10.1016/0006-8993(80)91265-2]

Weijl, N. I., Cleton, F. J., \& Osanto, S. (1997). Free radicals and antioxidants in chemotherapyinduced toxicity. Cancer Treatment Reviews, 23(4), 209-40. [DOI:10.1016/s0305-7372(97)90012-8] [PMID]

Xia, T., Kovochich, M., Brant, J., Hotze, M., Sempf, J., Oberley, T., ... \& Nel, A. E. (2006). Comparison of the abilities of ambient and manufactured nanoparticles to induce cellular toxicity according to an oxidative stress paradigm. Nano letters, 6(8), 1794-807. [DOI: 10.1021/nl061025k] [PMID]

Yadollahi, M., Farhoudian, S., Barkhordari, S., Gholamali, I., Farhadnejad, H., \& Motasadizadeh, H. (2016). Facile synthesis of chitosan/ $\mathrm{ZnO}$ bio-nanocomposite hydrogel beads as drug delivery systems. International Journal of Biological Macromolecules, 82, 273-8. [DOI:10.1016/j.ijbiomac.2015.09.064] [PMID]

Youssef, A. M., El-Nahrawy, A. M., \& Hammad, A. B. A. (2017) Sol-gel synthesis and characterizations of hybrid chitosanPEG/calcium silicate nanocomposite modified with $\mathrm{ZnO}$ NPs and (E102) for optical and antibacterial applications. International Journal of Biological Macromolecules, 97, 561-7. [DOI:10.1016/j.ijbiomac.2017.01.059] [PMID]

Youssef, A. M., El-Sayed, S. M., El-Sayed, H. S., Salama, H. H., \& Dufresne, A. (2016). Enhancement of Egyptian soft white cheese shelf life using a novel chitosan/carboxymethyl cel- lulose/zinc oxide bionanocomposite film. Carbohydrate Polymers, 151, 9-19. [DOI:10.1016/j.carbpol.2016.05.023] [PMID]

Zhang, M., Yan, H., Li, S., \& Yang, J. (2017). Rosmarinic acid protects rat hippocampal neurons from cerebral ischemia/ reperfusion injury via the Akt/JNK3/caspase-3 signaling pathway. Brain Research, 1657, 9-15. [DOI:10.1016/j.brainres.2016.11.032] [PMID] 
This Page Intentionally Left Blank 